\title{
Rain Attenuation in 5G Wireless Broadband Backhaul Link and Develop (IoT) Rainfall Monitoring System
}

\author{
Konstantinos Zarkadas ${ }^{1}$, George Dimitrakopoulos ${ }^{2}$ \\ Department of Informatics and Telematics, Harokopio University, Athens, Greece
}

\begin{abstract}
Climate change is the cause of more frequent and intense rainfall where they affect wireless communications because they cause severe weakening of the power of the emitted signal. These losses reduce network coverage and, therefore, system availability. The proposed solution is to integrate an Internet of Things (IoT) rainfall monitoring system where it will be able to collect real-time data on the height of rain that erupts in a particular place. This data will help areas where base stations install and the distance of the link that may need to be changed to reduce rainfall's harmful effects. So, the prediction of attenuation due to rain is an essential parameter in both terrestrial and satellite connections. The present study uses the ITU-R P 838 and ITU-R P 530 models to theoretically calculate losses in a 5G wireless broadband link with $99.9 \%$ link availability. This study conducts three frequency bands, $24 \mathrm{GHz}$, $28 \mathrm{GHz}$, and 38GHz, in Palo Alto, California. The travel distance is $5 \mathrm{~km}$, while the rainfall rate for the analyzed area is in zone $D$. The results show that the attenuations are proportional to the frequency, polarization, and rainfall rate.
\end{abstract}

Keywords-Rain attenuation; Internet of Things; wireless broadband

\section{INTRODUCTION}

Heavy rains occur more frequently, one of the many global warming [1], [2]. It is no longer a scenario that was to show its effects in the distant future. The situation is now difficult, and its first consequences are appearing. Studies have shown that global warming has a significant effect on precipitation. As early as 2011, the atmosphere's ability to retain water from water vapor increased by $7 \%$ for every $1^{\circ} \mathrm{C}$ of heating [3]. An essential factor in landslides in a geographical area is the seasonal period where the percentage of water is different, as revealed by research that has been studied [4]. More specifically, for the North American region, the rains during the summer months are more in the summer than in the winter. It also noted that energetic precipitation has been increasing both in frequency and rhythm since 1901.

Rainfall varies from year to year and is distinguished by the rate, duration, and amount of water that sometimes negatively impact crops, telecommunications, energy, and many other human activities and infrastructure. There are losses generated by electromagnetic waves transmitted during wireless communication in the telecommunications sector due to the absorption and scattering [5]. As a result, the coverage area and the efficiency of the wireless communication network are limited. The high frequencies in the microwave zone, millimeter-wave already used for 5G technology, are significantly affected by signal attenuation due to rain [6]. It studies that frequencies above $10 \mathrm{GHz}$ are significantly affected [7]. It is evident that climatic conditions significantly affect systems exposed or affected under the right weather conditions and cause malfunctions in the network. From the research so far, it cannot be accurately calculated that the amount of water will evolve on a rainy day. Even meteorological stations with their technological advancement in mathematical models may not have accurate data on the intensity or duration of rainfall or a storm. In recent years, smart meteorological stations have been created that calculates the intensity of the rain but not its height. So, the primary goal of the document is to design and implement a rainfall monitoring system. This solution promotes an IoT system that detects rainfall in real-time and provides information through a cloud service called Adafruit IO. This measured inferred whether the rate of rain would increase or not-this communication base on the MQTT protocol and the development of interfaces from the platform.

This article is structured as follows. Section 2 refers to the related work done so far and the theoretical background of rain attenuation. Section 3 shows the measurement setup and the theoretical measurements of the microwave link. In Section 4 present the experimental setup and analyze the IoT system of rain. The results will provide and discussed in Section 5. Finally, Section 6 contains concluding remarks and an outlook on future work potentials.

\section{THEORETICAL BACKGROUND}

\section{A. Related Work}

All Scientists and engineers are concerned about limiting or controlling the damage caused by heavy rainfall. They are developing search and alert applications for years with the help sensors and microcontrollers connect on the internet and transfer information to and from the user. In [8], an application is developed, which is essentially a meteorological station that informs about the meteorological changes such as the measurement of temperature, humidity, rainfall, carbon monoxide, altitude, and LPG in the environment. It consists of a microcontroller Arduino Mega where it connects to the individual sensors that, when stimulated, will give the message on the LCD screen. Besides, the device sends SMS to the user and informs them about the prices collected with a GSM module's help. In the end, the results compared with the data of the national meteorological company.

In [9], a system creates to control the rate of rainfall. It is a system that has the structural features of a rain gauge. It consists of a funnel that collects the water where it falls into a sensor-also connected to the EZ430-RF2500 where it contains the MSP430F2274 microcontroller and a CC2500 
transceiver for data transmission, which, in turn, is sent to another transceiver which is the central station connected to a laptop (Laptop computer unit) in C language in GUI graphical interface.

Even in [10], a system works as a rain gauge created; it consists of the detection unit with a sensor that works as a rain gauge. It consists of two parts, a funnel and a container that collects water. There is an Arduino Uno microcontroller that recovers data from the sensors. This system is even environmentally friendly as solar collector power it, and on days when there is no sunshine, the battery that has already charged use. There are three ways to communicate with the server: The station communicates through the $3 \mathrm{G}$ network with an Arduino Ethernet card and a 3G modem. The second through a $433 \mathrm{MHz}$ free band, which use for industrial or scientific purposes. Finally, it will be easier to use the GSM network with a GSM shield with an Arduino card.

Increasingly, the technological advancement of sensors in both field of application, cost, the size has contributed to the development of many constructions at both research and mechanical level. In particular, projects to detect floods or weather conditions in areas such as crops, livestock units, renewable energy units (e.g., solar parks), and even smart cities. Also, 3G and 4G technologies have further promoted the implementation of many such applications. Today, Internet of Things (IoT) technology is making its presence felt, which is greatly aided by the infrastructure, efficiency, and flexibility offered by 5G technology, equipped with high speeds, capacity, low latency, which offers reliability in telecommunications systems. Therefore, due to this technological boom and the reliability of wireless systems that should provide uninterruptedly, this article gives weight to studying the effects of rain on a point-to-point microwave connection. This connection has fifth technology features, and the data collected by calculations is from three different frequencies with an ordinary rainfall rate $(\mathrm{mm} / \mathrm{h})$. The difference with the research so far is that the rain detection systems have to do with developing a meteorological station or the creation exclusively of a rain meter where the rain rate calculates directly. This need in the present study was by developing an IoT system for calculating the amount of rain $(\mathrm{mm})$ that cleans itself every 1 second and informs in real-time about the rainfall course. Besides, a microcontroller uses where it collects information. The rain sensor and the help of a servo motor clean the flat surface to receive the next measurement. Finally, this system can interconnect the hardware through a cloud platform, accessible to the user. It will distinguish the sensor output and act immediately for any failures in terms of signal coverage or, in general, to analyze data on the availability of the broadband network.

\section{B. Background}

The signal attenuation calculates with appropriate models that help to calculate in theoretical attenuations [11]. The International Telecommunication Union (ITU) has helped facilitate international interconnection, spectrum sharing, and technical standards to ensure networks and technologies' smooth operation. Based on international recommendations (ITU-R P.838-3) [12], the specific attenuation of the $\gamma \mathrm{R}(\mathrm{dB} /$ $\mathrm{km})$ signal obtained by the rainfall rate $\mathrm{R}(\mathrm{mm} / \mathrm{h})$ from the following formula:

$\gamma R=K R a(d B / k m)$

The values $\mathrm{k}$ and a are coefficients that set depending on the operating frequency and the field polarization, defined by ITU-R P. 838 -3 in Table I.

The rate of rainfall comes in two ways, either from meteorological stations through individual measurements that calculate the average intensity per year, or from the categorization made by ITU - R. 837.1 [13] in acceptable climate zones in Table II.

In more detail, if a radio cluster with $99.99 \%$ availability is studied, it should be ensured that the connection will be available even at high rainfall rates that cause attenuation, with a probability of occurrence exceeding $0.01 \%$ of the time.

Therefore, after calculating the specific attenuation of the $\gamma \mathrm{R}(\mathrm{dB} / \mathrm{km})$ signal for horizontal and vertical polarization with the help of Table $\mathrm{I}$, the ground rate $\mathrm{r}$ factor is defined by the standard (ITU-R P 530.), should be calculated [14] and given by the following formula:

$r=\frac{1}{1+\frac{d}{d_{0}}}$

TABLE I. FACTORS K AND ACCORDING TO THE ITU-R P.838-3 STANDARD

\begin{tabular}{|l|l|l|l|l|}
\hline Frequency $(\mathrm{GHz})$ & $\mathrm{K}_{\mathrm{H}}$ & $\mathrm{a}_{\mathrm{H}}$ & $\mathrm{K}_{\mathrm{V}}$ & $\mathrm{a}_{\mathrm{V}}$ \\
\hline 20 & 0.09164 & 1.0568 & 0.09611 & 0.9847 \\
\hline 21 & 0.1032 & 1.0447 & 0.1063 & 0.9771 \\
\hline 22 & 0.1155 & 1.0329 & 0.1170 & 0.9700 \\
\hline 23 & 0.1286 & 1.0214 & 0.1284 & 0.9630 \\
\hline 24 & 0.1425 & 1.0101 & 0.1404 & 0.9561 \\
\hline 25 & 0.1571 & 0.9991 & 0.1533 & 0.9491 \\
\hline 26 & 0.1724 & 0.9884 & 0.1669 & 0.9421 \\
\hline 27 & 0.1884 & 0.9780 & 0.1813 & 0.90349 \\
\hline 28 & 0.2051 & 0.9679 & 0.1964 & 0.9277 \\
\hline 32 & 0.2778 & 0.9302 & 0.2646 & 0.8981 \\
\hline 34 & 0.3171 & 0.9129 & 0.3026 & 0.8834 \\
\hline 36 & 0.3580 & 0.8967 & 0.3427 & 0.8690 \\
\hline 38 & 0.4001 & 0.8816 & 0.3844 & 0.8552 \\
\hline
\end{tabular}

TABLE II. THE RATE OF RAINFALL IN (MM /H) DEEPENING ON THE PERCENTAGE OF TIME PER ZONE

\begin{tabular}{|l|l|l|l|l|l|l|l|l|l|l|}
\hline $\begin{array}{l}\text { Percentage } \\
\text { of time } \\
(\%)\end{array}$ & B & C & D & E & F & G & H & J & K & L \\
\hline 1.0 & 0.5 & 0.7 & 2.1 & 0.6 & 1.7 & 3 & 2 & 8 & 15 & 2 \\
\hline 0.3 & 2 & 2.8 & 4.5 & 24 & 45 & 7 & 4 & 13 & 42 & 7 \\
\hline 0.1 & 3 & 5 & 8 & 6 & 8 & 12 & 10 & 20 & 12 & 15 \\
\hline 0.03 & 6 & 9 & 13 & 12 & 15 & 20 & 18 & 28 & 23 & 33 \\
\hline 0.01 & 12 & 15 & 19 & 22 & 28 & 30 & 32 & 35 & 42 & 60 \\
\hline 0.003 & 21 & 26 & 29 & 41 & 54 & 45 & 55 & 45 & 70 & 105 \\
\hline
\end{tabular}


where $\mathrm{d}(\mathrm{km})$, the actual length of the path, while $\mathrm{d} 0$ is defined as follows:

$$
\left\{\begin{array}{c}
35 e^{-0.015 R_{0.01}} R_{0.01<100 \mathrm{~mm} / \mathrm{h}} \\
35^{-1.5} R_{0.01 \geq 100 \mathrm{~mm} / \mathrm{h}}
\end{array}\right.
$$

If $\mathrm{R} 0.01>100 \mathrm{~mm} / \mathrm{h}$, place the value $100 \mathrm{~mm} / \mathrm{h}$ in place of R0.01 The length of the current path calculated:

$d_{\text {eff }}=r * d(\mathrm{~km})$

Thus, the total value of the losses is calculated as follows:

Horizontal polarization

$L^{H}=\gamma_{R 0.01}^{H} * d_{e f f}(d B)$

Vertical polarization

$L^{V}=\gamma_{R 0.01}^{V} * d_{\text {eff }}(d B)$

\section{Measurement Setup}

1) Description of area: The selected area is Palo Alto (37 $2613 \mathrm{~N}-1220748 \mathrm{~W})$, located northwest of Santa Clara County, California, USA. It locates in the center of Northern California, and the choice of this location is not accidental. Heavy rains in northern California expect to increase in the coming years [15]. Also, the geographical area belongs to zone D, according to the ITU - R. standard 837.1.

2) Experimental data: The scenario concerns a wireless broadband link with Line of Sight (LOS), where the route length is $5 \mathrm{~km}$. Both the transmitter and the receiver operate at 24, 28, and $38 \mathrm{GHz}$. These frequencies are ideal. After all, they work in the $5 \mathrm{G}$ spectrum because they have lower oxygen uptake rates [16]. The equipment is iPASOLINK NEC VR4 based on fifth-generation (5G) technical specifications Fig. 1. This equipment is already used by telecommunications providers as a reliable wireless backhaul solution to support a fifth-generation (5G) network. It consists of an external ODU type IAG unit with channel bandwidth up to $112 \mathrm{MHz}$, low power consumption - $13 \sim 38 \mathrm{GHz}$ : $42 \mathrm{dBm}$ (30\% lower than the current version), modulation 2048 QAM, with a capacity of $1 \mathrm{Gbps}$ and a bandwidth of $112 \mathrm{MHz}$, elements that are equally compatible with NR 5G Fig. 2.

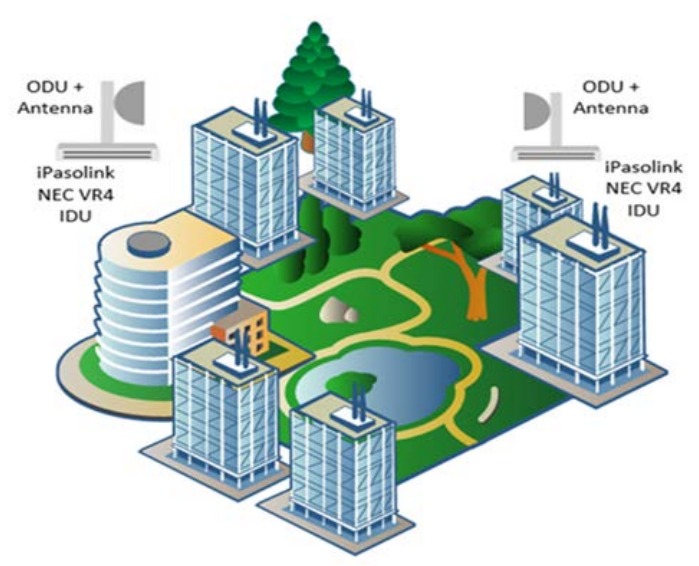

Fig. 1. Location of Wireless Broadband Backhaul Link.

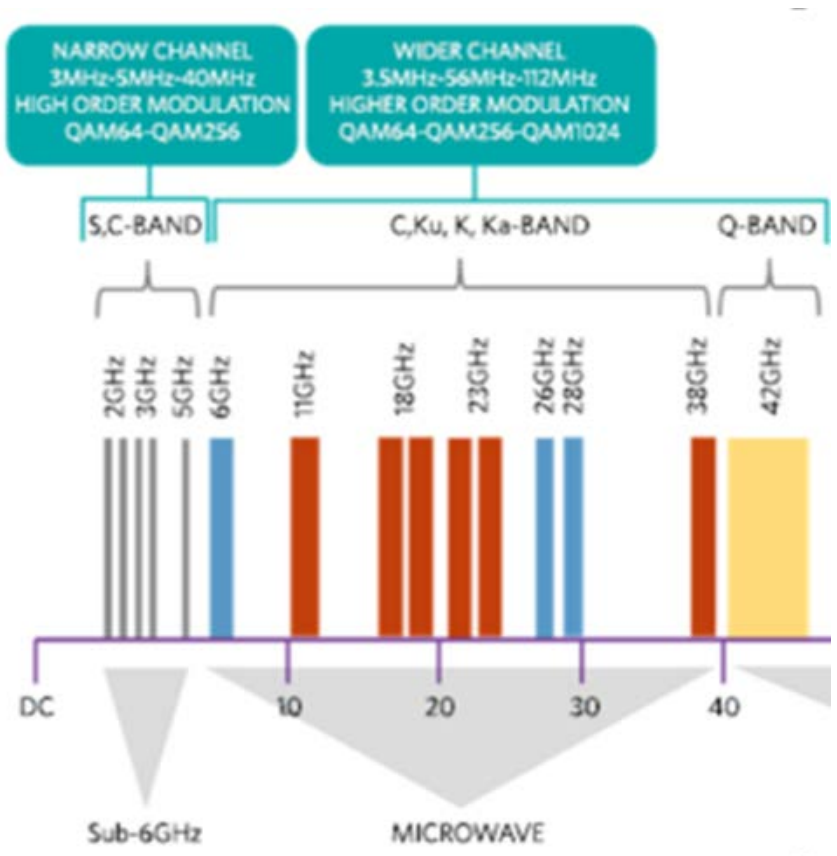

Fig. 2. Characteristics of Microwave Wireless Backhaul [17].

\section{Theoretical Measurements}

At this point, rainfall attenuation with coupling availability of $99.99 \%$ is calculated, with a distance of $5 \mathrm{~km}$ for each of three operating frequencies:

- $38 \mathrm{GHz}$

\section{Horizontal polarization}

According to Table II, the coefficients $\mathrm{K}$ and $\alpha$ at $38 \mathrm{GHz}$ receive the following values.

$\mathrm{KH}=0.4001, \alpha=0.8816$

With the help of relation (1) the losses per km are:

$\gamma_{R 0.01}^{H}=K_{H} * R_{0.01}^{a}=0,4001 * 19^{0,8816}=5,36\left(\frac{\mathrm{dB}}{\mathrm{km}}\right)$

Vertical polarization

Similarly, as before, the losses for vertical polarization are calculated

$\gamma_{R 0.01}^{V}=K_{V} * R_{0.01}^{a}=0,3844 * 19^{0,8552}=4,76\left(\frac{\mathrm{dB}}{\mathrm{km}}\right)$

To calculate the total losses of horizontal and vertical polarization, the losses per $\mathrm{km}$ (as done earlier) and the current deff path's length must be measured. It is useful because it shows that along with the link, there is heterogeneity of meteorological conditions (e.g., more torrential rain elsewhere, not elsewhere), it is always less than the actual length. The current deff path calculate by multiplying the factor $r$ by the actual length $\mathrm{d}$.

After first calculating the sufficient path length through rain, for $\mathrm{R}_{0.01}<100 \mathrm{~mm} / \mathrm{h}$ :

$d_{0}=35 e^{-0,015 * R 0,01}=35 e^{-0,015 * 19}=26,32$ 
Thus, the reduction factor $\mathrm{r}$ :

$r=\frac{1}{1+\frac{d}{d_{0}}}=\frac{1}{1+\frac{5}{26,32}}=0,84$

Therefore, the length of the active path can now set:

$d_{\text {eff }}=r * d=0,84 * 5=4,2 \mathrm{~km}$

So the total losses for each polarization are calculated separately:

Horizontal polarization

$L_{R 0,01}^{H}=\gamma_{R 0,01}^{H} * d_{e f f}=5,36\left(\frac{d B}{k m}\right) * 4,2(k m)=22,51 d B$

Vertical polarization

$L_{R 0,01}^{V}=\gamma_{R 0,01}^{V} * d_{e f f}=4,76\left(\frac{d B}{k m}\right) * 4,2(k m)=20 d B$

- $24 \mathrm{GHz}$

Horizontal polarization

According to Table II, the coefficients $\mathrm{K}$ and $\alpha$ at $24 \mathrm{GHz}$ take the following values. With the help of relation (1) the losses per km are:

$\gamma_{R 0.01}^{H}=K_{H} * R_{0.01}^{a}=0,142 * 19^{1,01}=2,77\left(\frac{\mathrm{dB}}{\mathrm{km}}\right)$

Vertical polarization

Similarly, as before, the losses for vertical polarization are calculated

$\gamma_{R 0.01}^{V}=K_{V} * R_{0.01}^{a}=0,140 * 19^{0,95}=2,29\left(\frac{\mathrm{dB}}{\mathrm{km}}\right)$

To calculate the total losses of horizontal and vertical polarization, the losses per $\mathrm{km}$ (as was done earlier) and the current deff path's length must be measured-the current deff path calculated by multiplying the factor $\mathrm{r}$ by the actual length $d$.

After first calculating the sufficient path length through rain, for $\mathrm{R}_{0.01}<100 \mathrm{~mm} / \mathrm{h}$ :

$d_{0}=35 e^{-0,015 * R 0,01}=35 e^{-0,015 * 19}=26,32$

Thus, the reduction factor $\mathrm{r}$ :

$r=\frac{1}{1+\frac{d}{d_{0}}}=\frac{1}{1+\frac{5}{26,32}}=0,84$

Therefore, the length of the active path can now set:

$d_{\text {eff }}=r * d=0,84 * 5=4,2 \mathrm{~km}$

So, the total losses for each polarization are calculated separately:

Horizontal polarization

$L_{R 0,01}^{H}=\gamma_{R 0,01}^{H} * d_{e f f}=2,77\left(\frac{d B}{k m}\right) * 4,2(k m)=11,63 d B$
Vertical polarization

$L_{R 0,01}^{V}=\gamma_{R 0,01}^{V} * d_{\text {eff }}=2,29\left(\frac{d B}{k m}\right) * 4,2(k m)=9,61 d B$

- $28 \mathrm{GHz}$

Horizontal polarization

According to Table II, the coefficients $\mathrm{K}$ and $\alpha$ at $28 \mathrm{GHz}$ take the following values.

With the help of relation (1) the losses per km are:

$\gamma_{R 0.01}^{H}=K_{H} * R_{0.01}^{a}=0,20 * 19^{0,96}=3,37\left(\frac{\mathrm{dB}}{\mathrm{km}}\right)$

Vertical polarization

Similarly, as before, the losses for vertical polarization are calculated

$\gamma_{R 0.01}^{V}=K_{V} * R_{0.01}^{a}=0,19 * 19^{0,92}=2,86\left(\frac{\mathrm{dB}}{\mathrm{km}}\right)$

To calculate the total losses of horizontal and vertical polarization, the losses per $\mathrm{km}$ (as was done earlier) and the current deff path's length must be measured. The current deff path calculate by multiplying the factor $r$ by the actual length $d$.

After first calculating the sufficient path length through rain, for $\mathrm{R}_{0.01}<100 \mathrm{~mm} / \mathrm{h}$ :

$d_{0}=35 e^{-0,015 * R 0,01}=35 e^{-0,015 * 19}=26,32$

Thus the reduction factor $\mathrm{r}$ :

$r=\frac{1}{1+\frac{d}{d_{0}}}=\frac{1}{1+\frac{5}{26,32}}=0,84$

Therefore, the length of the active path can now set:

$d_{\text {eff }}=r * d=0,84 * 5=4,2 \mathrm{~km}$

So the total losses for each polarization are calculated separately:

Horizontal polarization

$L_{R 0,01}^{H}=\gamma_{R 0,01}^{H} * d_{e f f}=3,37\left(\frac{d B}{k m}\right) * 4,2(k m)=14,15 d B$

Vertical polarization

$L_{R 0,01}^{V}=\gamma_{R 0,01}^{V} * d_{e f f}=2,86\left(\frac{d B}{k m}\right) * 4,2(k m)=12,01 d B$

\section{EXPERIMENTAL SETUP}

\section{A. System Architecture}

IoT is a device system that interconnects via the Internet. It does with the help of many platforms and protocols on the market. The development of this rainfall detection system (IoT) aims to inform the user about the course of the precipitation, as it is an essential factor for the degree of intensity with which the rain erupts in a certain period (per hour or minute) [18]. The system analyzed consists of a low-cost microcontroller (ESP32) with integrated Wifi and dual-function Bluetooth 
(Fig. 3). This connection to all the sensors and many parts of circuits such as (power supplies, analog inputs-outputs, digital outputs, and breadboard). Its primary function is to collect the sensors' data, process them, and inform about its condition through the Adafruit IO service with the internet unit's help. Also used is a SUNKEN rain sensor with dimensions of $13 \mathrm{x}$ 8.4 x 1.3 inches and a Miuzei SG90 9G micro servo motor (Fig. 4).

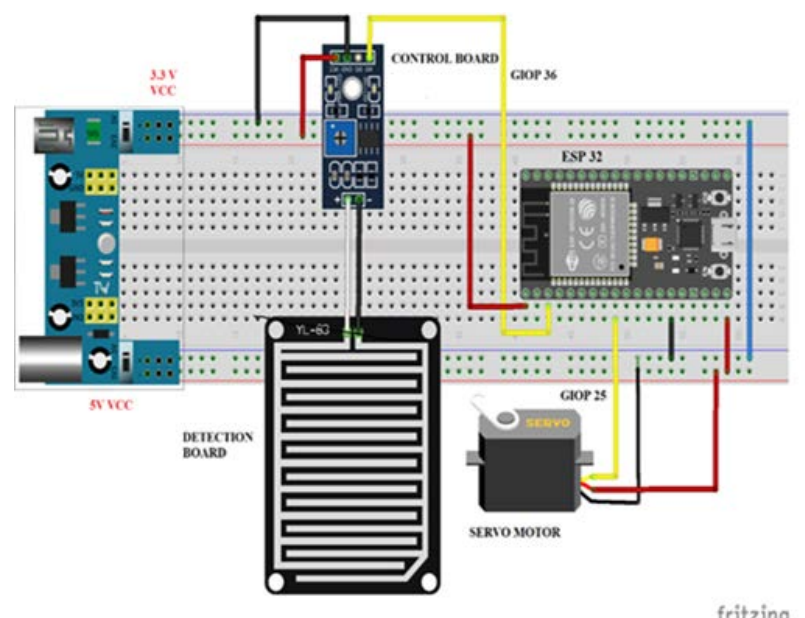

Fig. 3. Simulation of Project.

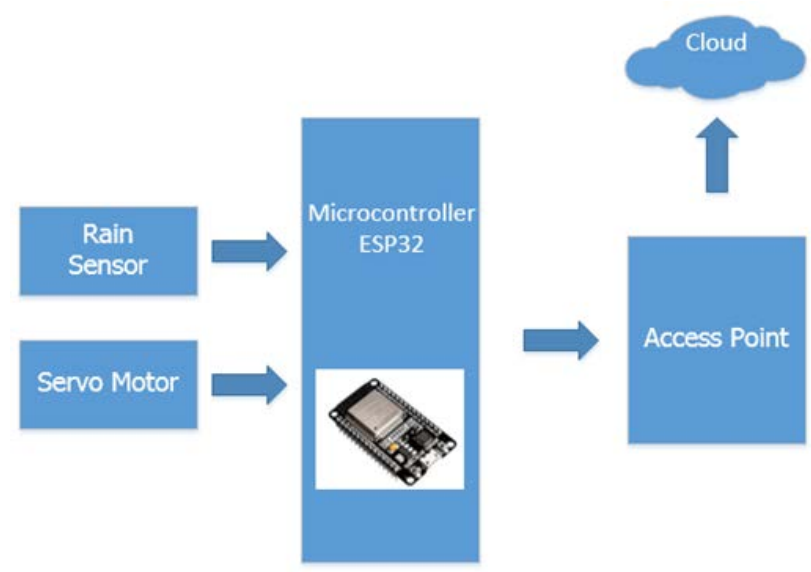

Fig. 4. Block Diagram of Projects.

\section{B. Input System}

This system works by receiving data from a rain sensor, where the engine activates after a specific range of values. The output data obtained through ESP 32, where analyzed below:

- Input Data of Rain Sensor

This device uses to control rain or flood in various applications. It included a detection board, a control board where the sensor's sensitivity is regulated-his logic based on a variable resistance. The sensor is a resistor that shows less resistance when wet and more resistant when dry. When there is no water drop on the surface, then the resistance increases, and so at the output, there is a high voltage according to $\mathrm{V}=\mathrm{IR}$ (Ohm's Law). Correctly, the rain sensor can be used both as a digital input, i.e., distinct values of 0 or 1 can characterize it. (e.g., a switch that can be on (Off / Off) or it can use as an analog input, i.e., it can be characterized as from a price range (e.g., a potentiometer where it can get a price range depending on where it turned). So when there is an analog device, the reading of an analog value with ESP 32 allows us to measure different voltage levels between $0 \mathrm{~V}$ and $3.3 \mathrm{~V}$, where this can translate into 0 to 1023 integers. The range is determined by the built-in ADC, which in the case of ESP 32, is ADC 10 bit $(2 \wedge 10=1024)$. The specifications of rain sensor set out in the Table III.

\section{- Servo motor}

Servo motors are small devices that have a shaft protruding on their outer casing. It is a device that can rotate an axis from 0 to 180 or 360 degrees. He could change the angle (move) if a coded signal sents. As long as this signal is present on the Servo input line, it will keep its axis in a specific position. When the signal changes, it causes the servo to change the angle of the shaft. This device is used for many projects, such as controlled aircraft, cars, and robotics. In this case, the engine uses as an analog output.

- Process System

Make clear to the real-time user that the values collected by the microcontroller have set up, a scale showing the total value of the analog value range set by Table IV is displayed, depending on the amount of water falling on the panel. This table has created with the help of a cylindrical tube graded in millimeters [9]. Finally, it should note that the panel place at a slope of 40 , which results in the expulsion of a large amount of water more significant than $50 \mathrm{~mm}$.

- $\quad$ Micro controller esp 32

The ESP32-DEVKIT V1 is an MCU Wi-Fi + BT + BLE unit with a wide range of applications, from low-power network sensors to the most demanding tasks such as voice coding, music streaming, and MP3 decoding. Two CPU cores can control individually, and the CPU clock frequency is adjustable from $80 \mathrm{MHz}$ to $240 \mathrm{MHz}$. The controller has a data rate of up to $150 \mathrm{Mbps}$ and an output power of $20.5 \mathrm{dBm}$ on the antenna to ensure wireless transmission. Its integration with Bluetooth and WIFI makes it flexible for many applications. The controller can connect digital input/output units and analog inputs/outputs, useful for many applications. These units are nothing more than electronic data that, when excited, send a signal to the controller, then it gives the corresponding command with which the user programs it. At this stage, the program code writes in MicroPython language.

TABLE III. SPECIFICATIONS OF THE RAIN SENSOR

\begin{tabular}{|l|l|}
\hline Pin & Description \\
\hline Voltage Rating & $5 \mathrm{~V}$ \\
\hline GND & Negative power source. \\
\hline Power supply voltage & $3.3-5 \mathrm{~V}$ \\
\hline Sensor Board Size & $54 \mathrm{~mm} \times 40 \mathrm{~mm}$ \\
\hline Shield PCB Size & $30 \mathrm{~mm} \times 16 \mathrm{~mm}$ \\
\hline Sensitivity Adjustment & Clockwise is more sensitive. \\
\hline
\end{tabular}


TABLE IV. RULE BASE SYSTEM

\begin{tabular}{|l|l|}
\hline Analog value & Direct reading gauge $(\mathbf{m m})$ \\
\hline $980-890$ & 5 \\
\hline $890-780$ & 10 \\
\hline $780-670$ & 20 \\
\hline $670-580$ & 30 \\
\hline $580-450$ & 40 \\
\hline $450-400$ & 50 \\
\hline
\end{tabular}

- Software IDE

In this study, use Thonny, where is the Python Integrated Development Environment (IDE) used. It is especially useful for code entry. It has a built-in error detection program. This editor also allows to program the ESP32 and ESP8266 boards with MicroPython and is compatible with Windows, Mac OS $\mathrm{X}$, and Linux. MicroPython came from the Python 3 language and developed to program microcontrollers and electronic devices connected to it. While MicroPython is not yet as wellknown as $\mathrm{C}$ and $\mathrm{C}++$ for electronic device scheduling, it is slowly becoming popular with more and more microcontrollers and IDE. It is also effortless to use, and its interface makes it very easy for a novice user. It also features a read-evaluateprint (REPL). This feature helps the user connect to the board and execute it directly without compiling it. In the present phase, the interpreter, MicroPython (ESP32), and the corresponding USB prey that the board has connected to select the code to load on it select.

\section{- Adafriut IO}

Adafruit.IO is a cloud service - this means that it does not need to be managed by the user. This platform uses to connect to the Internet. To store and retrieve data, but can do much more than that. Adafruit.Io can handle and visualize many data streams. The platform environment is quite user-friendly (Fig. 5). Also, the Adafruit online platform works with many microcontrollers (including ESP32) and, with the support of the MQTT protocol, allows easy and secure management of IoT systems.

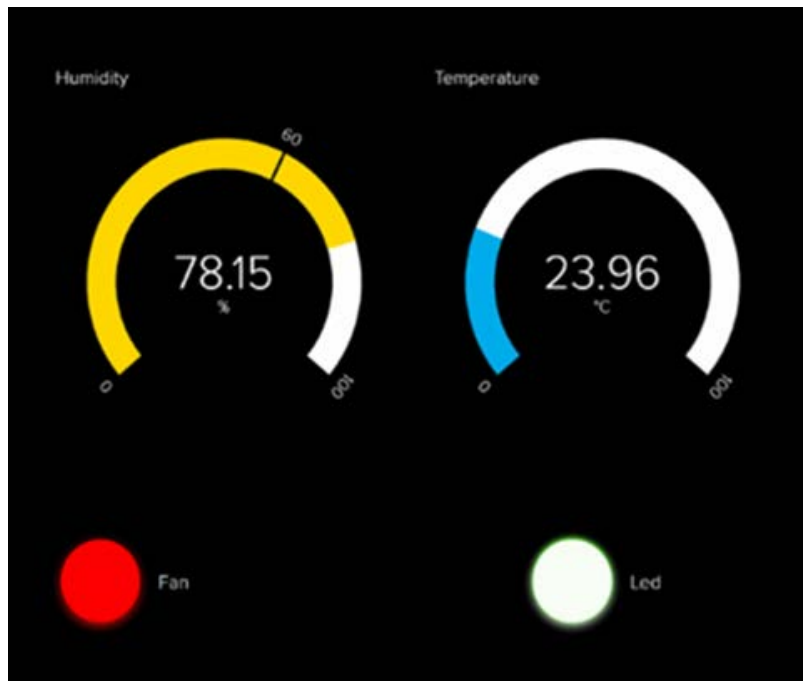

Fig. 5. Interface Adafruit IO.

\section{Working of IoT based Rainfall Monitoring System}

The construction shows that the rain height's size can place in a base station, a residential complex, lighting columns, and different environments (urban or rural areas) (Fig. 6). The basic idea is to inform the user in real-time via a local network from the platform. An ESP32 device can play the role of an Access Point or a Station. The data output thus passes to the Adafruit IO platform via the MQTT protocol.

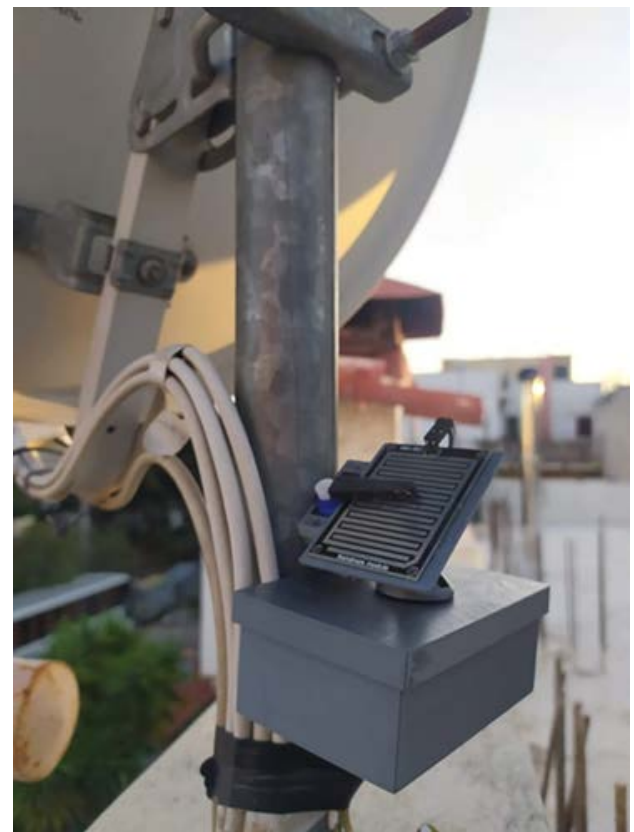

Fig. 6. IoT based Rainfall Monitoring System with 3D Case.

The sender is the (publisher), and the recipient is the (subscriber). Both do not come into direct contact with each other. However, a third party (MQTT broker) directs the publisher's messages to any endpoint, that is, the subscriber. The following Fig. 7 shows the IoT system architecture, where the sensor is located on the transmitter and through the MQTT broker sends the appropriate messages to the monitor that controls them in real-time.

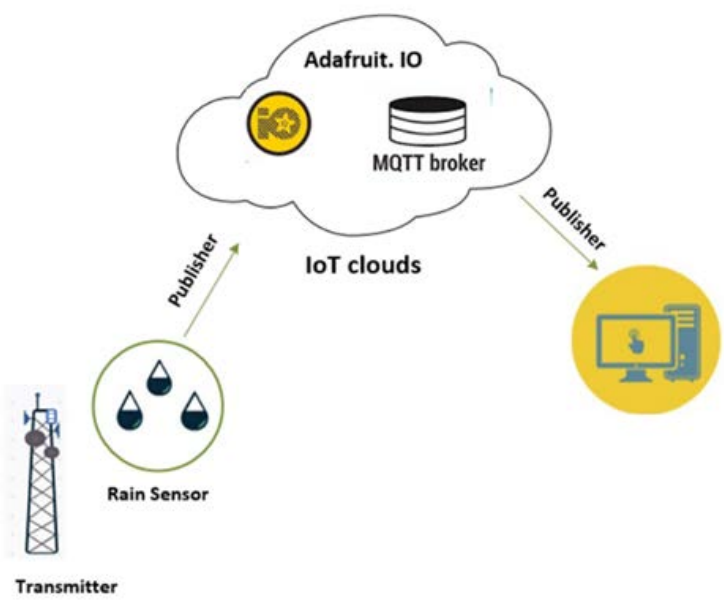

Fig. 7. MQTT Broker Network Architecture in Wireless Broadband. 
The board has programmed so that the dry surface indicates the value of 1023 . The values that can be displayed come from many experimental tests with a cylindrical tube graded in millimeters. There are no other values from one point onward because the rain panel is placed at a slope of $40^{\circ}$, resulting in not enough water to remain when it falls on it due to gravity.

\section{RESUlts AND Discussion}

\section{A. Theoretical Measurement Results}

Theoretical weather calculations on wireless communication systems would be more straightforward if there were no rain or humidity effects. As it is known from the bibliography so far, the rain can weaken the radio waves' power depending on its rhythm and operation frequency. The present study presents the scenarios presented at Palo Altobased on three different frequencies of $24 \mathrm{GHz}, 28 \mathrm{GHz}$, and $38 \mathrm{GHz}$, where they are ideal for 5G wireless broadband connections.

From the theoretical measurements made, the rainfall attenuation calculates with a coupling availability of $99.9 \%$, with a distance of $5 \mathrm{~km}$ for each of the three operating frequencies (Fig. 8). It found that the lower the operating frequency, the lower the radio signal's attenuation, and that different loss values are presented based on polarization. Horizontal polarization is more prone to damping than vertical polarization. As raindrops increase in size, they get more extended in the horizontal direction. They, therefore, will attenuate horizontal polarization more than vertical polarization. The shape of the drops explains this as they fall from the atmosphere. In particular, during the fall, they change shape showing the tendency to distribute their volume, mainly along the horizontally polarized electric field. In this regard, the field interaction with the droplet is more critical in horizontal polarization than in vertical. Therefore, the scenario where it is less affected by rainfall is the operation at $24 \mathrm{GHz}$. The frequency range from $24 \mathrm{GHz}$ and above offers low latency and high data rate [19]. Such a wireless connection will continue to provide high-quality services to users, even in severe weather conditions.

The specific attenuation depends on the value of the frequency. As the frequency increases, so do the specific attenuation based on the international recommendations (ITUR P.838-3) [12].

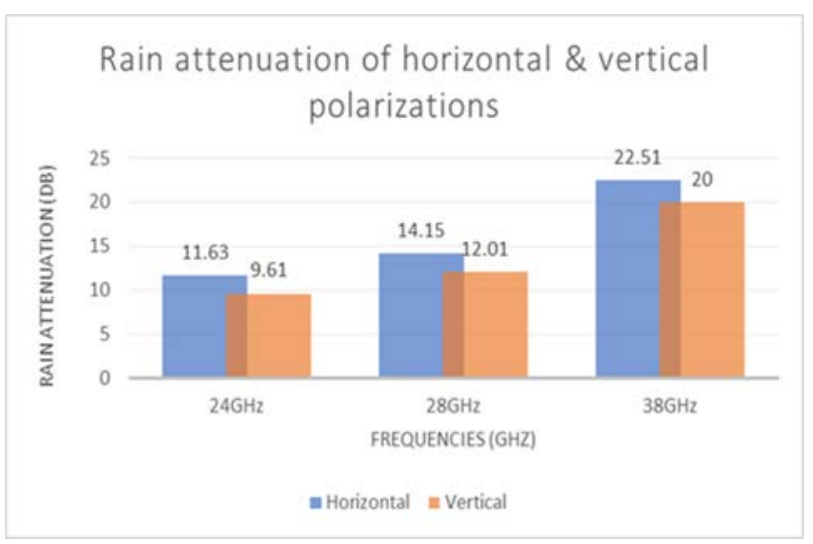

Fig. 8. Rain Attenuation of Horizontal and Vertical Polarizations.

\section{B. Esp 32 Thonny IDE Output}

As studied in the scenario that took place, the parameter of the rate of the rainfall $\mathrm{R}(\mathrm{mm} / \mathrm{hr}$ ) is critical because the original size that characterizes it is the height of the rain, where it measures in millimeters. In the theoretical measurements that followed, the rate was the same due to its geographical location. At present paper, an IoT system develops, which will present in real-time the rainfall in the standards of a scale that create for the display of the sizes. The planning divided into three sections:

In the first one, the required libraries have defined, the calls of the Pin, PWM, I2C, ADC classes, and the initializations of the analog input for the rain sensor and the initializations for the analog output, which is the servo motor (Fig. 9).

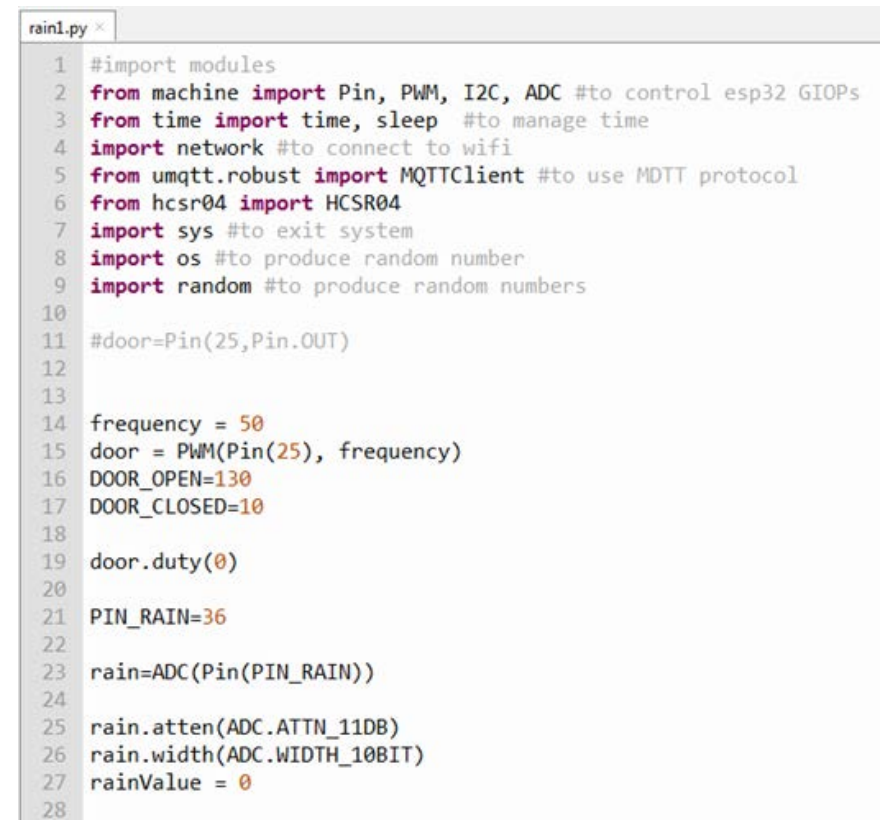

Fig. 9. Libraries and Initialization of Variables.

In the second part of the code is defined as the communication MQTT client publisher, with the MQTT broker (server), where this, in turn, communicates with client subscribers. A Client can be both a publisher and a subscriber. The sensor's measurement is in line 119, and the value of the measurement is sent with the Send_Data () function on line 122. Essentially the line of code that sends the measurement to the interface is line 86. The above line sends the information str (rainValue) through the client created above (line 48), "mqtt_feedname1". Specifically, the path, "io.adafruit.com/kostaszar/feeds/rain". Rain is the name of the feed on the interface side, i.e., the value rainValue will send to the interface block connected to the feed rain.

The third and last show all those commands that are the program's central structure and are the ones that have the central role. It is essentially a repetition where it is checked every 10 seconds for the values it takes. If the values listed in the platform interface are below 800 , then the most significant amount of water is collected in the panel, so there is a need to clean where after two seconds, the rain sensor is ready to receive water again for the next recording. Within these two 
seconds, the engine has made two $180^{\circ}$ turns. With a drop of 5 $\mathrm{mm}$, the water drop, the program settings so that the range of values it can get is between 980 and 890 . Then the $10 \mathrm{~mm}$ of water corresponds to $890-780$ and the $20 \mathrm{~mm}$ of water in 780 - 670. The next Price range is 670 - 580 were with $30 \mathrm{~mm}$ of water. Finally, with $40 \mathrm{~mm}$ of water space, precipitation is 580 - 450, and with $50 \mathrm{~mm}$ of water, a value appears between 450 400.

Every element presents a size or an output to a feed. Each feed is a communication channel between ESP 32 (client) and Adafruit (broker). Dashboards can place the icons (blocks) by the developer. In this analysis, a rain gauge defines, from which the value displayed appears at a specific time, and the user can easily see it.

\section{CONCLUSION}

As studied for the $24 \mathrm{GHz}, 28 \mathrm{GHz}$, and $38 \mathrm{GHz}$ frequency bands, damping due to rain may seem vital because they create network coverage problems. Especially at $38 \mathrm{GHz}$, the damping for horizontal polarization is $22.51 \mathrm{~dB}$. At $28 \mathrm{GHz}$, the damping is less by $8.36 \mathrm{~dB}$, while at $24 \mathrm{GHz}$, the losses are smaller than the other two frequencies where they reach 11.63 $\mathrm{dB}$ for horizontal polarization.

In the era of the fifth generation of wireless broadband networks in urban areas due to the increased coverage and capacity requirements, the mobile communication networks distributed in cells. Where within a macro cell, there may be many smaller ones to serve more broadband services. The phenomenon of attenuation will be more evident in rural areas because, in addition to the prevailing weather conditions, the distance that the electromagnetic wave must travel as it travels taken into account. In contrast to urban areas where the distances are short, it seems that the $24 \mathrm{GHz}$ frequency is ideal from the other two, although they are suitable for the development of fifth-generation networks.

Thus, mobile companies' ultimate solution will be to continually update the weather conditions prevailing in a base station or a wireless broadband backhaul installation-ideal for the places where the climate is changing rapidly and is affected by heavy rainfall. According to this document, sensor systems will be useful because they can interconnect with the World Wide Web and other devices that will allow them to exchange data and provide the required information.

\section{REFERENCES}

[1] Chen, C., Harvey, J.A., Biere, A., \& Gols, R. (2019). Rain downpours affect survival and development of insect herbivores: the specter of climate change? Ecology, 100.

[2] Yin, J., Gentine, P., Zhou, S., Sullivan, S.C., Wang, R., Zhang, Y., \& Guo, S. (2018). Large increase in global storm runoff extremes driven by climate and anthropogenic changes. Nature Communications.

[3] Trenberth, K.E. (2011). Changes in precipitation with climate change.
[4] Easterling, D.R., K.E. Kunkel, J.R. Arnold, T. Knutson, A.N. LeGrande, L.R. Leung, R.S. Vose, D.E. Waliser, and M.F. Wehner, 2017: Precipitation change in the United States. In: Climate Science Special Report: Fourth National Climate Assessment, Volume I [Wuebbles, D.J., D.W. Fahey, K.A. Hibbard, D.J. Dokken, B.C. Stewart, and T.K. Maycock (eds.)]. U.S. Global Change Research Program, Washington, DC, USA, pp. 207-230, doi: 10.7930/J0H993CC.

[5] Singh, H., Bonev, B., Petkov, P.Z., \& Kumar, R.R. (2018). A Novel Approach for Predicting Attenuation of Radio Waves caused by Rain. 2018 2nd IEEE International Conference on Power Electronics, Intelligent Control and Energy Systems (ICPEICES), 1193-1198.

[6] M. Ghanim, M. Alhilali, J. Din and H. Y. Lam, "Rain Attenuation Statistics over 5G Millimetre Wave Links in Malaysia," 2018 5th International Conference on Electrical Engineering, Computer Science and Informatics (EECSI), Malang, Indonesia, 2018, pp. 266-269.

[7] Shayea, I., Rahman, T.A., Azmi, M.H., \& Islam, M.R. (2018). Real Measurement Study for Rain Rate and Rain Attenuation Conducted Over $26 \mathrm{GHz}$ Microwave 5G Link System in Malaysia. IEEE Access, 6, 19044-19064.

[8] M. I. Haque, A. H. MD. Shatil, A. N. Tusar, M. Hossain and M. H. Rahman, "Renewable Powered Portable Weather Update Station," 2019 International Conference on Robotics,Electrical and Signal Processing Techniques (ICREST), Dhaka, Bangladesh, 2019, pp. 374-377.

[9] O.Omoruyi, S. N. John, O. Chinonso, O. Robert, A. A. Adewale and K. O.Okokpujie, "Wireless Sensor Network for Rainfall Measurement Using a Tipping Bucket Rain Gauge Mechanism," 2017 International Conference on Computational Science and Computational Intelligence (CSCI), Las Vegas, NV, 2017, pp. 740-744.

[10] O.Manzombi, E. M. Dogo and N. I. Nwulu, "Design and Implementation of a Wireless Patient Health Monitoring System," 2019 International Artificial Intelligence and Data Processing Symposium (IDAP), Malatya, Turkey, 2019, pp. 1-6.

[11] Kestwal, M.C., Joshi, S., \& Garia, L.S. (2014). Prediction of Rain Attenuation and Impact of Rain in Wave Propagation at Microwave Frequency for Tropical Region (Uttarakhand, India).

[12] Recommendation ITU-R P.838-3 (03/2005), "Specific attenuation model for rain for use in prediction methods", P Series, Radiowave propagation.

[13] Recommendation ITU-R PN.837-1 (08/1994), "Characteristics of precipitation for propagation modelling”, P Series, Radiowave propagation.

[14] Recommendation ITU-R P.530-9 (02/2001), "Propagation data and prediction methods required for the design of terrestrial line-of-sight systems, P Series, Radiowave propagation.

[15] Pathak, T.B.; Maskey, M.L.; Dahlberg, J.A.; Kearns, F.; Bali, K.M.; Zaccaria, D. Climate Change Trends and Impacts on California Agriculture: A Detailed Review. Agronomy 2018, 8, 25.

[16] Rappaport, T. S., Murdock, J. N., \& Gutierrez, F. (2011). State of the art in $60-\mathrm{GHz}$ integrated circuits and systems for wireless communications. Proceedings of the IEEE, 99(8), 1390-1436. [5958173]. https://doi.org/10.1109/JPROC.2011.2143650.

[17] Ahmadi, Sassan. 5G NR: Architecture, Technology, Implementation, and Operation of 3GPP New Radio Standards. N.p.: Academic, 2019. Print.

[18] Brouwer, C., Goffeau, A. and Heibloem, M. (1985) Irrigation Water Management: Training Manual No. 1-Introduction to Irrigation. FAO, Rome.

[19] Ancāns, Guntis \& Bobrovs, Vjaceslavs \& Ancans, Arnis \& Kalibatiene, Diana. (2017). Spectrum Considerations for 5G Mobile Communication Systems. Procedia Computer Science. 104. 509-516. 10.1016/j.procs.2017.01.166. 